\title{
Golden Age of Radio Astronomy
}

\author{
R. D. Ekers ${ }^{1}$ \\ CSIRO Astronomy and Space Science - ATNF \\ Sydney, NSW, Australia \\ E-mail:ron.ekers@csiro.au
}

\begin{abstract}
One of the most important events in twentieth century astronomy was the birth of radio astronomy. For the first time ever astronomers were able to view the Universe in a region of the electromagnetic spectrum outside the narrow optical window. These early discoveries were usually unexpected and often unpredicted. They were initially made by individual scientists from other disciplines who built very unconventional "telescopes". This included the development of aperture synthesis in Cambridge, Australia and The Netherlands.
\end{abstract}

These early pioneers discovered a plethora of cosmic phenomena that revolutionized our knowledge of the Universe. I will explore some of the paths which lead to these discoveries in radio astronomy and conclude with lessons learned as we move into the next "Golden Age".

ISKAF2010 Science Meeting - ISKAF2010

Assen, the Netherlands

June 10-14 2010

\footnotetext{
$1 \quad$ Speaker
} 


\section{The Ages of radio astronomy}

- The beginning - first light: 1930s

Jansky, and later Reber, opened a new field of astronomical research using radio waves.

- The heroic golden age: 1950s - 1960s

Many innovative instrumental developments and discoveries including three Nobel Prize winning discoveries.

- The decadent golden age: 1970s - 1980s

Many astronomers are using the new radio telescopes with many exciting results but little instrumental innovation is occurring.

- The decline: 1990 - 2000

Radio astronomy is eclipsed as observations at many new wave length bands (IR, UV, X-ray, $\gamma$-ray) are made possible by the space observatories. The development of new optical instrumentation and large optical telescopes is now progressing faster than radio techniques.

- The new golden age: $2010 \ldots .$.

The SKA and its various pathfinders have rejuvenated radio astronomy in many countries with new innovative developments across all radio frequencies.

\section{Beginning of radio astronomy}

Woody Sullivan's [1] definitive new history of early radio astronomy provides a comprehensive and insightful study of the emergence of this new branch of astronomy.

\subsection{Karl Jansky's discovery}

In the course of trying to identify the source of interference to trans-Atlantic telephone communications Karl Jansky, working at the Bell Telephone Laboratory, discovered cosmic radio emission in 1933 [2]. An unexpected source of noise (Jansky's Cosmic Hiss) was peaking each day but the peak signal arrived 4 min earlier each day and Jansky realised that it must have extraterrestrial origins. Reaction from Bell Labs was underwhelming: "so faint not even interesting as a source of radio interference!" Once he had determined that the interference was "of extraterrestrial origin," there was little support from the Bell Telephone Laboratory to further pin down the location in space. It was also not accepted by the astronomical community at the time and Jansky died in 1950 before the importance of his discovery was appreciated. Jansky's daughter - Moreau Jansky-Parsons remembered that her father said his boss made him change "emission from interstellar space" to "emission apparently from interstellar space". 


\subsection{Grote Rebers' challenge}

In 1933 Karl Jansky had reported the discovery of radio emission from the centre of the Galaxy. Grote Reber thought this was so interesting that someone should follow up. At the time he was a 22 year old engineering graduate specializing in electronics and communication, he later commented: "Professional astronomers showed little interest so I consulted with myself and built a dish."

\subsection{The ascendance of low frequency radio astronomy}

In 1937 Reber built his 32' parabolic dish in Wheaton, Illinois and started looking for radio emission. First he looked at shorter wavelengths than Jansky since the predictions for thermal radio emission would make it stronger at shorter wavelengths [3]. Nothing was seen at $3300 \mathrm{MHz}$ or $900 \mathrm{MHz}$ but finally at $160 \mathrm{MHz}$ he eventually detected cosmic static similar to that seen by Jansky but stronger at longer wavelengths. This radio emission had to be non-thermal but there was no concept of non-thermal astronomical emission at the time. It was not until 1950 that the synchrotron radiation mechanism was understood [4].

Grote Reber made the first radio map of sky but had great difficulty getting it published. Confirming Jansky's observations and making a radio map of the Galaxy was not serendipitous but the discovery of the non-thermal emission was an unexpected discovery.

\subsection{Cygnus A, strongest radio source in the sky}

In 1946 Hey found a source of radio emission with intensity varying on time scales of seconds to minutes [5]. He correctly concluded that the source must be small diameter. This was the first "radio star". But what was it? There was no optical counterpart. Was the whole galactic plane was made of such stars? Note that at this time there was no theory linking the diffuse galactic emission to cosmic rays.

\subsection{Cliff interferometer}

In 1946 at Dover Heights near Sydney a telescope was constructed on the cliff to measure the interference between the direct waves and those reflected by the sea (a Lloyd's mirror). This cliff interferometer was built to to locate the origin of the solar radio emission [6] and to identify the radio stars [7]. The idea of a cliff interferometer came from multiple path interference seen in ship-borne radar and which was used to improve positional information. Bolton and his colleges were able to use it to measure positions accurately enough to identify three of the strongest of the mysterious discrete sources of radio emission which were thought to be radio stars.

One was the Crab nebula, the remnant of a star that the Chinese had seen explode 900 years ago. The other two were an even greater surprise. Centaurus A and Virgo A were discrete sources like Cygnus A but they had conspicuous bright optical identifications which were galaxies - not stars! They were both galaxies, far outside our own milky way but undergoing such a violent explosion that they were among the brightest objects in the radio sky. Bolton et al had to hedge on the extragalactic origin to get the paper published. This discovery, with some help from the now very enthusiastic optical astronomers at Mt Palomar in the US, lead to the eventual 
identification of the strongest of all the radio sources, Cygnus A. It was found to be a very faint galaxy so incredibly distant that it was immediately obvious that these new radio telescopes were probing the most distant reaches of the universe. A new field of astronomy was born.

As a result of their tremendous luminosity, radio galaxies could be observed from very great distances, far beyond the reach of the best optical telescopes of the time. Radio source number counts based on early Cambridge surveys led Martin Ryle to argue in favour of an evolving universe [8], although later observations showed that even though the Cambridge data were badly corrupted by confusion and the analysis was mathematically incorrect [9], Ryle's conclusions were right - the Universe was evolving.

\section{$2.621 \mathrm{~cm}$ hydrogen line}

In 1945 van de Hulst predicted the possible existence of a radio spectral line due to the spin flip transition of neutral hydrogen [10]. It was detected by Ewen \& Purcell at Harvard in 1950. The discovery involved international collaboration - a US discovery confirmed by Dutch \& Australian groups. The American and Dutch results were published in the same issue of Nature [11] [12]. These were followed by a brief note in the same issue from Pawsey mentioning that Christiansen and Hindman had also detected the line in Australia.

See Sullivan's Chapter 16 [1] for an in-depth commentary which discusses the difficult war time period and the initial scepticism about its detectability. These stories should give impetus and some lessons for the groups currently searching for the same line from the epoch of re-ionisation.

\section{Aperture synthesis}

\subsection{The beginnings of aperture synthesis}

The first published suggestion that it would be possible to synthesise an image of the radio sky by measuring a range of Fourier components was made by McCready, Pawsey and Payne-Scott in 1946 [13]. However, this technique was impractical with a cliff interferometer, and was not suitable for imaging solar bursts which were strongly variable in both time and frequency. The first observations using a range of Fourier components measured with an interferometer with moveable elements were made at the Cavendish Laboratory [14] [15] [16].

\subsection{Earth rotation synthesis images}

In June 1961 radio astronomers at the Cavendish Laboratory in Cambridge, UK, used $4 \mathrm{C}$ aerials operating at $178 \mathrm{MHz}$ to make a radio source survey of the North Pole region [17]. Computations and graphical display used EDSACII which was the first use of a digital computer for radio astronomy imaging. Comparable images at low frequencies are only just now being made again with the new generation of telescopes. This was 7 years after Christiansen [18] first demonstrated the earth rotation synthesis with an observation of the quiet sun using an array of small dishes in Australia. 
However, the Australian group took many months to calculate the Fourier transforms by hand so the method was considered impractical at the time.

The Cambridge group went on to build the One-Mile Telescope [19] in 1962 and the 5km Telescope in 1971. Martin Ryle was awarded the Nobel Prize in 1974 "For his observations and inventions, in particular for the aperture synthesis technique".

\subsection{Benelux Cross: 1958}

The Benelux Cross [20] was a joint Netherlands - Belgium project initiated by Professor Jan Oort to use the radio astronomy source counts for cosmology. International coordination was through an agreement with the OEEC (Organisation for European Economic Cooperation), now the OECD. Key radio engineers from round the world were brought in to design the telescope. It was very ambitious with $100 \times 30 \mathrm{~m}$ dishes plus one $70 \mathrm{~m}$ dish to add short spacing information. Operating at $21 \mathrm{~cm}$ with baselines to $1.5 \mathrm{~km}$ to have sufficient resolution to avoid confusion. The science goals for the Benelux Cross were described by Oort at an OECD Symposium in 1961. The primary goal was to have enough sensitivity and resolving power to study the early universe through source counts.

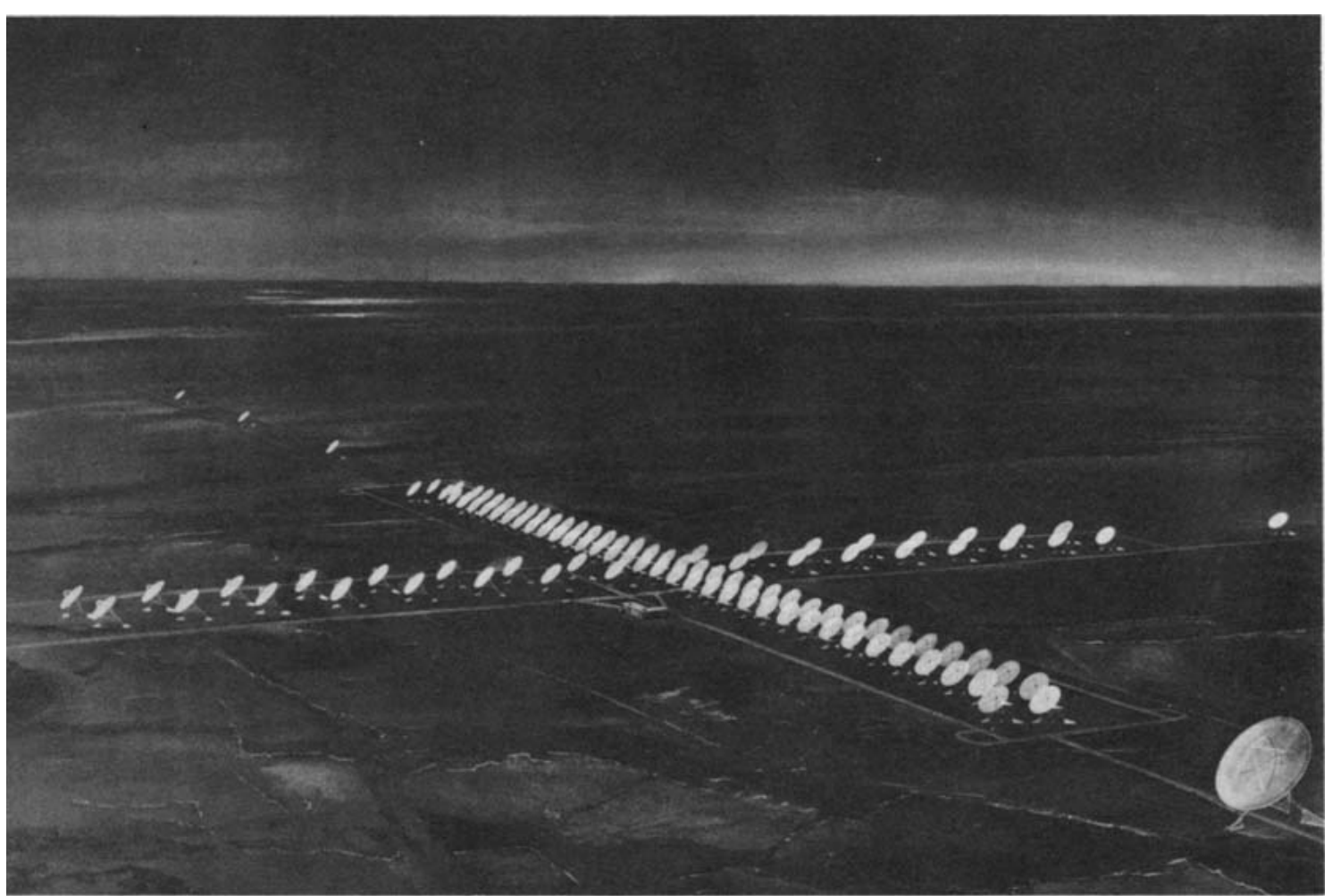

Figure 1. Artists impression of the Benelux Cross (Ben Hooghout 1963)

\subsection{Westerbork: 1970}

By 1964 the Benelux Cross project had become a purely Dutch affair. The design was drastically modified under the influence of Jan Hogbom, a recent $\mathrm{PhD}$ graduate from Cambridge, and Chris Christiansen, from CSIRO in Sydney. The Benelux Cross 
was transformed into the Westerbork Synthesis Radio Telescope (WSRT) which combined aspects of aperture synthesis using moveable elements and earth rotation synthesis with the grating array concepts. The WSRT has $14 \times 25 \mathrm{~m}$ dishes. Four moveables and the other ten are at fixed equal spacings. This provided a number of identical, and hence redundant, spacings and these redundant spacings were the key to the realisation that self-calibration could be extended to arbitrary source distributions.

\section{Nobel Prize discoveries}

Table 1 lists the 10 astronomical discoveries which have resulted in Nobel Prizes. In Figure 2 I have plotted these discoveries against the discovery date and a subjective indication of the relative scale of the instrument or research group involved. Five of these ten discoveries have been made at radio wavelengths, a remarkable fraction of all Nobel Prizes in astronomy. It is quite clear from Figure 2 that the role of Big Science facilities in making discoveries increases in importance with time [21].

Table1: Nobel Prizes for astronomy

\begin{tabular}{|l|l|l|l|}
\hline Prize & Experiment & Subject & Laureates \\
\hline 1936 & 1912 & Cosmic Rays & Victor Franz Hess (shared) \\
\hline 1974 & 1960 & Aperture Synthesis & Sir Martin Ryle \\
\hline 1974 & 1967 & Pulsars & Antony Hewish \\
\hline 1978 & 1965 & CMB & Arno A. Penzias, Robert W. Wilson \\
\hline 1983 & 1931 & Stellar Evolution & Subrahmanyan Chandrasekhar \\
\hline 1983 & 1950 & Chemical Elements & William Alfred Fowler \\
\hline 1993 & $1974-78$ & Gravitational Radiation & $\begin{array}{l}\text { Russell A. Hulse, Joseph H. Taylor, } \\
\text { Jr. }\end{array}$ \\
\hline 2002 & 1987 & Cosmic Neutrinos & $\begin{array}{l}\text { Raymond Davis, Jr., Masatoshi } \\
\text { Koshiba }\end{array}$ \\
\hline 2002 & 1962,70 & Cosmic X-rays & Riccardo Giacconi \\
\hline 2006 & 1989 & CMB & John C. Mather, George F. Smoot \\
\hline
\end{tabular}




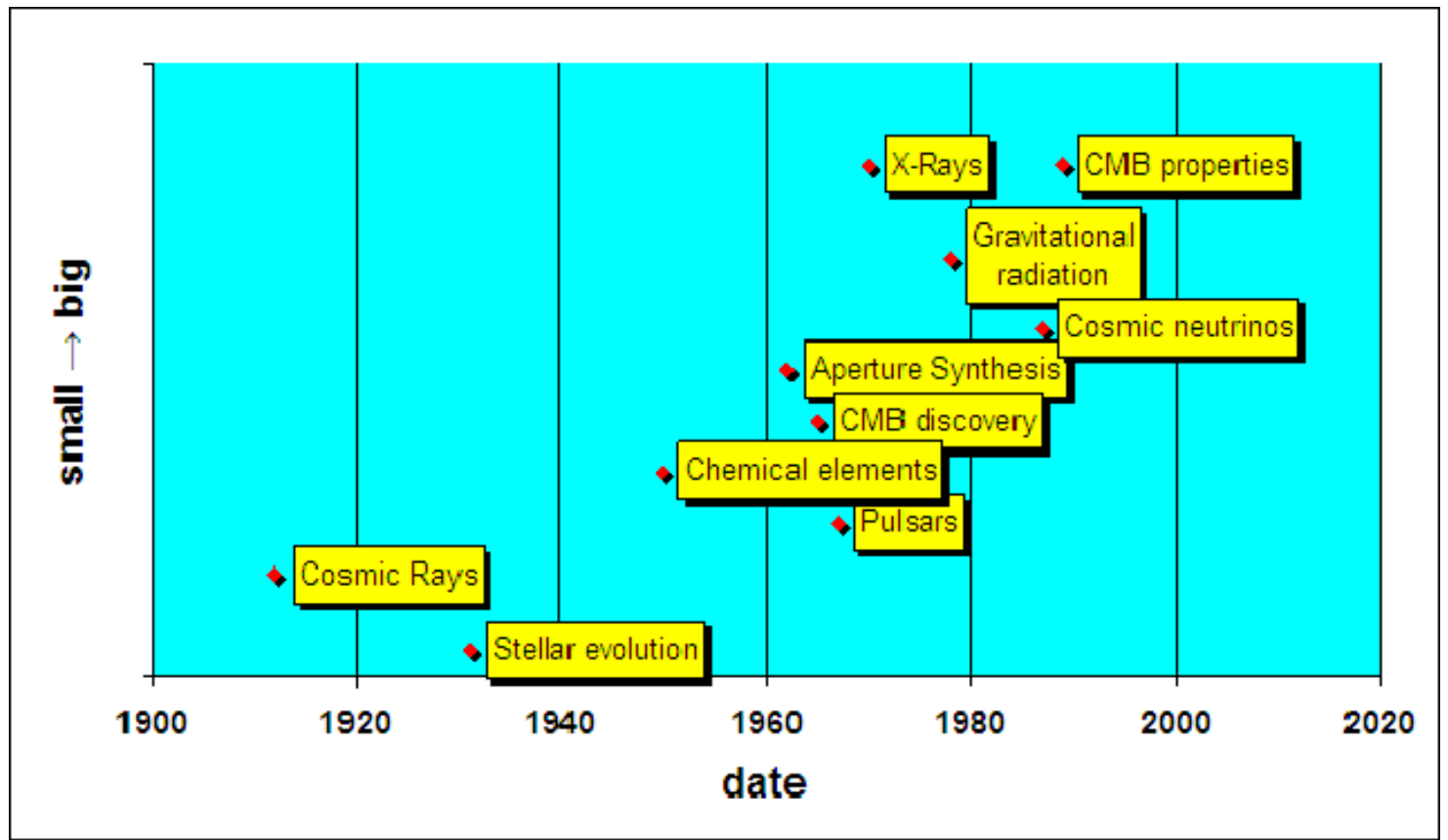

Figure 2: Nobel Prizes in astronomy vs. date of discovery with an indication of the relative scale of the experiment [21]

\subsection{Pulsar discovery Cambridge: 1967}

In 1967 Tony Hewish and his student Jocelyn Bell made a serendipitous discovery of pulsars [22] at Cambridge with a telescope built to study interplanetary scintillation. The 1974 Nobel Prize was awarded to Tony Hewish "For his decisive role in the discovery of pulsars".

\subsection{Cosmic microwave background: 1965}

Some thirty years later, in the same Bell Laboratories where Jansky had discovered cosmic radio emission, Arno Penzias and Bob Wilson discovered the three degree cosmic microwave background (CMB) while trying to understand the apparent losses in a radio antenna also designed to support trans-Atlantic telephone communication [23]. They were awarded the 1978 Nobel Prize for the discovery of the Big Bang radiation. This was a serendipitous observation of a predicted phenomenon. Bob Dicke's experiment to search for $\mathrm{CMB}$ radiation based on his prediction was already in progress.

\subsection{Gravitational radiation: 1974}

The 1993 Noble Prize was awarded to Joe Taylor and Russell Hulse for the verification of Einstein's prediction of gravitational radiation [24]. This is a classic example of the scientific method of prediction and observation. 


\subsection{COBE - the microwave background: 1989}

The Nobel Prize in Physics for 2006 was awarded jointly to John C. Mather, NASA Goddard Space Flight Center, Greenbelt, MD, USA, and George F. Smoot, University of California, Berkeley, CA, USA, "for their discovery of the blackbody form [25] and anisotropy [26] of the cosmic microwave background radiation".

\section{Other Discoveries in Radio Astronomy}

The beginning of radio astronomy provides excellent examples of discoveries made by exploring the unknown. Wilkinson et al [27] included a tabulation of the key discoveries in radio astronomy since the beginning of the field in 1933 to 2000. Figure 3 (a) plots these discoveries against time, comparing the discoveries made with special purpose instruments with those made on the larger general user facilities. It is clear that the number of discoveries made with special purpose instruments has declined with time. Figure 3 (b) shows that serendipitous discoveries are more prevalent at the inception of a new branch of science.

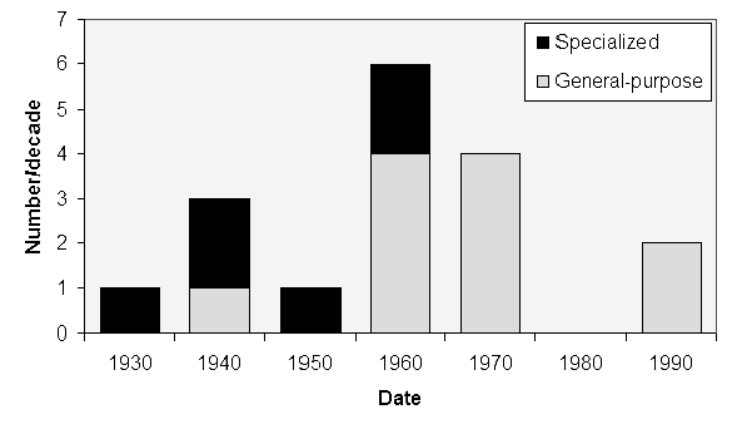

(a) Type of instrument

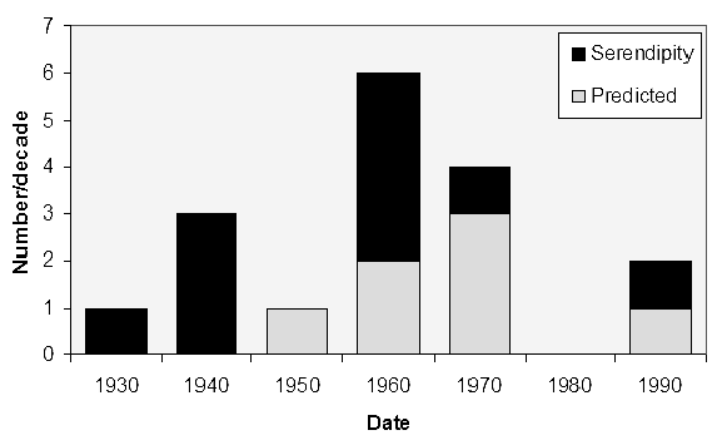

(b) Predicted v Serendipity

Figure 3: Key Discoveries in Radio Astronomy from [27]

The number of discoveries made with special purpose instruments has declined with time as discussed by Harwit [28]. There are a number of possible reasons:

- It is easier to use existing general purpose instruments than build special purpose ones.

- The people who can build special purpose instruments leave the field.

- The discoveries which can be made without the sensitivity of the big general purpose instruments have already all been made.

\section{VLA New Mexico}

\subsection{Building the VLA}

In 1965 a proposal to construct the VLA was submitted to the US National Science Foundation (NSF). The VLA construction commenced in 1972 and it was formally 
inaugurated in 1980. The VLA has been the most productive ground based telescope ever built, in both its publications and citations.

\subsection{VLA science}

What a radio telescope was built for is almost never what it is known for. Almost invariably, the discoveries in Table 1 of Wilkinson et al [27] were not, often could not have been, in the minds of the designers of these telescopes. For example, Jodrell Bank was built to study meteor trails, Arecibo to study the ionosphere, and the WSRT to do weak source counts. The 1967 VLA funding Proposal submitted to NSF listed the key scientific drivers (8 pages) and this included: radio galaxies, quasars, cosmology. It also listed other science which may benefit: planets, galactic studies, and $21 \mathrm{~cm}$ Hydrogen line.

The author compiled a list of the actual science being done with the VLA between 1980 and 1991. Table 2 shows that the VLA, which is one of the most productive astronomical telescopes of all time, spent only a quarter of its time during its initial decade of operation on the key science drivers listed in the funding proposal.

Table 2. Distribution of VLA Science 1980 - 1991

\begin{tabular}{|l|c|l|c|}
\hline Topic & $\begin{array}{c}\text { Observing } \\
\text { Time (\%) }\end{array}$ & Topic & $\begin{array}{c}\text { Observing } \\
\text { Time (\%) }\end{array}$ \\
\hline Stars & 16 & Interstellar medium & 4 \\
\hline Galaxies & 14 & Cosmology & 4 \\
\hline Radio Galaxies & 13 & Molecules & 3 \\
\hline Quasars & 9 & Galactic Centre & 3 \\
\hline Star formation & 9 & VLBI & 3 \\
\hline Solar system & 6 & Pulsars & 2 \\
\hline AGN & 5 & X-ray, etc & 1 \\
\hline Supernovae & 4 & Astrometry & 1 \\
\hline
\end{tabular}

Items in bold were the key science drivers in the 1967 funding proposal

\section{Exponential growth in science}

Harwit [28] showed that most important discoveries in astronomy result from technical innovation. The discoveries peak soon after new technology appears, and usually within 5 years of the technical capability. Instruments used for discoveries are often built by the observer. He also noted that new astronomical phenomena are more frequently found by researchers trained outside astronomy. It had already been well established that most scientific advances follow technical innovation in other areas of science. In 1960 de Solla Price [29] applied quantitative measurement to the progress of science (scientometrics) and reached the conclusion that most scientific advances follow laboratory experiments. His analysis also showed that the normal mode of growth of science is exponential. 


\subsection{Growth of radio telescopes}

Figure 4 plots the sensitivity of telescopes used for radio astronomy since the discovery of extra-terrestrial radio emission in 1940. It has been exponential with an increase in sensitivity of $10^{5}$ since 1940 , doubling every three years. Also in this case we can see particular radio telescope technologies reaching ceilings and new technologies being introduced e.g., the transition from huge single dishes to arrays of smaller dishes in the 1980s.

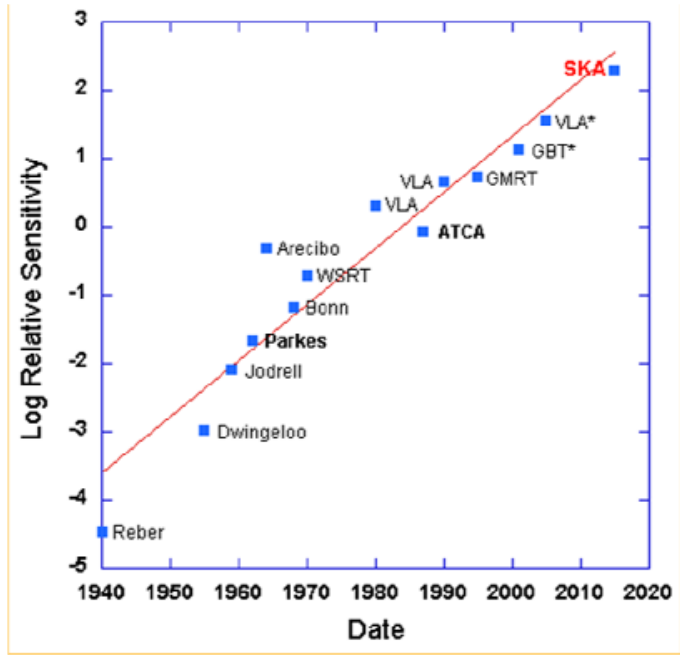

Figure 4: Radio Telescope Sensitivity vs. time. Points are the relative continuum sensitivity when the telescopes were built, or after major upgrades. VLA* is the EVLA upgrade.

SKA is the proposed sensitivity for a telescope which has not yet been built.

\subsection{How to maintain exponential growth?}

If the improvement in sensitivity has reached a ceiling the rates of new discoveries will decline and the field will become uninteresting and die out [21] [29] [30]. On the other hand, if we can shift to new technology or find new ways to organize our resources the exponential increase in sensitivity can continue. Do we have such new technology to continue the exponential improvement? In radioastronomy the combination of transistor amplifiers and their large scale integration into complex systems which can be duplicated inexpensively provides one of the keys for change. The other key technology is the computing capacity to apply digital processing at high bandwidth thereby realizing processes such as multiple adaptive beam formation and active interference rejection in ways not previously conceivable. Finally, the move to international facilities such as the proposed SKA will also be needed to avoid the resource ceiling.

\subsection{From little science to big science:}

Exponential growth cannot continue indefinitely without hitting the ceiling on cost or available resources to continue. As discussed in the previous section, sometimes technical innovation comes to the rescue but de Solla Price [29] [30] also recognized the important role played by the transition from Individual Researcher to Institute to National Facility and, finally, to International Facility, each step removing a resource limitation ceiling. He coined the terms 'little science' and 'big science' to describe the two extremes. 
- Institutional Facilities are built to enable research on a scale which no individual can afford.

- National Facilities are built to enable research on a scale which no single institute can afford.

- International Facilities are built to enable research on a scale which no single nation can afford.

While the progression clearly involves an increasing big science component, this doesn't mean the small science role has to disappear, and as discussed in the following sections, it is important that it doesn't disappear.

\section{Managing scientific research}

In the 1950s Irving Langmuir, General Electric, (Nobel Prize for Chemistry, 1932) noted that you can't plan to make discoveries but you can plan a work environment which increases the chance of discovery. He argued that you need to encourage individual freedom to explore, provide opportunities for discussion in an open environment and encourage contacts outside the field. He also argued that it is necessary to avoid the over protection of information, over management, and lack of time to pursue other ideas.

\section{Lessons learned}

What can we learn from our past experience? Large scale facilities make it possible to do projects which no single group, or even nation can afford. Large scale facilities may also be able to make the unexpected discoveries but we must be very aware of the pitfalls.

\subsection{What does this mean for the SKA?}

We need to maximise flexibility in the design to follow up on the unexpected discoveries. This means that we must use key science drivers carefully and not allow them to constrain the design. Remember that the main role of a key science driver is to promote the known science that can be done and to quantify specifications.

To reduce the cost we must seek ways for the multiple reuse of expensive components, such as simultaneous multiple beams or spectral bands which use the same collection areas.

We need to exploit the areas of fastest changes in technology by designing the signal processing hardware in such a way that the major purchases can be made late in the project. Begin the software development early; the SKA will inevitably be the largest software project in astronomy, and there is no Moore's Law escalator to help cover software costs. 
We must maintain the technical expertise in community. The experience in the USA over the past two decades during which technology development in many of the traditional university departments systematically fell away as resources were directed to the national facilities, should act as a warning.

Pathfinders are necessary to explore technical and scientific issues but they must be able to adapt to change to optimise the final instrument.

\subsection{Challenges for large scale facilities}

- It is important to get the interaction with the user community right by accessing the diversity of ideas and broadening the knowledge pool available. We also need the support from the community of astronomers at many levels so it is important to keep them happy and productive. Representative committees are needed but tend to be conservative and risk averse. I have always thought it better for a TAC to advise the director, not to make decisions.

- Continued investment is needed to maintain forefront facilities and to keep the best scientists and engineers engaged in the project.

- You need to reward and keep the innovative instrumentalists and programmers but current citation based advancement processes do not naturally do this.

- It is important to maintain innovative research groups and technology development in the community.

- A process is needed to support the non-standard projects. These may be high risk and benefit only a small group but they may also seed future innovation. The priesthood model with expert users providing the interface to the general astronomy community may be effective.

- How to provide rapid response to unexpected events.

\subsection{How do we go forward?}

We must build new large scale facilities and we should understand why governments fund large scale infrastructure. We need to learn how to collaborate in international facilities without extinguishing individual innovation. We need a user community to set specifications to test predictions for specific science cases but we also need big picture system scientists and engineers who understand how to build instruments since their innovation will drive our new endeavours.

The excitement of these new instruments is not the old questions they will answer but the new questions they will raise. 


\section{References}

[1] W.T. Sullivan, Cosmic Noise, Cambridge University Press, 2009.

[2] K. Jansky, 1933, Electrical Disturbances Apparently of Extraterrestrial Origin, Proc. I.R.E., 21, 387.

[3] G. Reber, Cosmic Static, Proc IRE 28, p68-70, 1940.

[4] H. Alvén, N. Herlofson, Cosmic radia tion and radio stars, Phys Rev 78, p616, 1950.

[5] J.S. Hey, S,J. Parsons, J.W. Phillips, Fluctuations in cosmic radiation at radio frequencies, Nature 158, p234, 1946.

[6] J. L. Pawsey, R. Payne-Scott, L. McCready, Radio-Frequency energy from the sun, Nature 157, p158-9, 1946.

[7] J. G. Bolton, G. J. Stanley, and O. B. Slee, 1949, Positions of Three Discrete Sources of Galactic Radio-Frequency Radiation, Nature, 164, 101.

[8] M. Ryle, and P. A. G. Scheuer, 1955, The Spatial Distribution and the Nature of Radio Stars, Proc. Roy Soc. A230, 448.

[9] K. I. Kellermann and J. V. Wall, 1987, Radio Source Counts and their Interpretation in Observational cosmology; Proceedings of the IAU Symposium No. 124, 1987, Reidel, p545.

[10] H.C. Van de Hulst, Radiogolven uit het wereldruim, Nederlandsch Tijdschrift v. Natuurkunde 11, p210-221,1945. English translation available in W Sullivan, Classics in Radioastronomy, Reidel 1982

[11] H.I. Ewen, E.M. Purcell, Radiation from galactic hydrogen at 1420 Mc/s, Nature 168, p356, 1951.

[12] C.A. Muller, J.H. Oort, The interstellar hydrogen line at $1420 \mathrm{Mc} / \mathrm{s}$ and an estimate of the galactic rotation, Nature 168, p357-358, 1951.

[13] L.L. McCready, J.L. Pawsey, R. Payne-Scott, Solar radiation at radio frequencies and its relation to sun spots, Proc Roy Soc A190, p357-375, 1947.

[14] H.M. Stanier, Distribution of radiation from the undisturbed sun at a wave-length of $60 \mathrm{~cm}$, Nature $165, \mathrm{p} 354-355,(1950)$

[15] K.E. Machin, Distribution of radiation across the solar disk at a frequency of $81.5 \mathrm{Mc} / \mathrm{s}$, Nature 167, p889-89, 1951.

[16] P.A. O'Brien, The distribution of radiation across the solar disk at metre wave-lengths, MNRAS 113, p597-612 (1953)

[17] M. Ryle, A.C.Neville, A radio survey of the North Polar region with a 4.5 minute of arc pencilbeam system, MNRAS 125, p39, 1962.

[18] W. Christiansen, J. Warburton, The distribution of radio brightness over the solar disk at a wavelength of 21 centimetres. III The quiet sun - two-dimensional observations, Aust J Phys, Vol 8, p474-486, 1955.

[19] Ryle, M.; Hewish, A., The synthesis of large radio telescopes, MNRAS 120, p220, 1960. 
[20] History of the Benelux Cross Project http://www.astron.nl/radio-observatory/public/historywsrt/benelux-cross-antenna-project/benelux-cross-antenna-project

[21] R.D. Ekers, Big and Small, in Accelerating the Rate of Astronomical Discovery - SPS5, IAU GA, Rio de Janeiro, Brazil, August 11-14, 2009.

[22] A. Hewish et al, 1968, Observation of a Rapidly Pulsating Radio Source, Nature 217, 709.

[23] A.A. Penzias and R. W. Wilson, 1965, A Measurement of Excess Antenna Temperature at 4080 Mc/s, Ap.J., 142, 419.

[24] R. A. Hulse and J. H. Taylor, 1975, Discovery of a Pulsar in a Binary System, ApJ, 195, L51.

[25] J.C. Mather et al, Measurement of the Cosmic Microwave Background Spectra by the COBE FIRAS Instrument, ApJ 420, p439-444, 1994.

[26] G.F. Smoot et al, Structure in the COBE differential microwave radiometer first-year maps, ApJ 396. , p. L1-L5, 1992.

[27] P.N Wilkinson, K.I. Kellermann, R.D. Ekers, J.M. Cordes, T.J.W. Lazio, The exploration of the unknown, New Astronomy Reviews, Vol. 48, Issue 11-12, p. 1551-1563, 2004.

[28] M. Harwit, Cosmic Discovery - The Search, Scope \& Heritage of Astronomy, Basic Books Inc., New York 1981.

[29] D.J. de Solla Price, Little science, big science, Columbia University Press, 1963

[30] D.J. de Solla Price, Little science, big science - and beyond, Columbia University Press, 1986 